\title{
Impact analysis of mobile phone electromagnetic radiations on human electroencephalogram
}

\author{
SUMAN PATTNAIK ${ }^{1, *} \mathbb{D}$, BALWINDER SINGH DHALIWAL $^{2}$ and S S PATTNAIK ${ }^{3}$ \\ ${ }^{1}$ IKG Punjab Technical University, Jalandhar, Punjab, India \\ ${ }^{2}$ Guru Nanak Dev Engineering College, Ludhiana, India \\ ${ }^{3}$ National Institute of Technical Teachers' Training \& Research, Chandigarh, India \\ e-mail:sumanbhagat1199@gmail.com; bsdhaliwal@ymail.com; profshyampattnaik@gmail.com
}

MS received 4 October 2018; revised 13 February 2019; accepted 28 March 2019; published online 6 May 2019

\begin{abstract}
The experimental study of electromagnetic interference of mobile phone radiations on brain waves is a contemporary research area and the ever-increasing use of mobile phones make it more imperative to explore the problem area in detail. Electromagnetic signal from mobile phones operating in Global System for Mobile (GSM) and wide band code division multiple access (WCDMA), has been considered in this paper and their interference impacts have been analyzed on the human electroencephalogram (EEG). The impact on brain waves i.e., delta, theta, alpha, beta and gamma waves are analyzed in five modes namely ideal mode i.e., when mobile phone is not in use, transmission mode and the receiving modes of second generation $(2 \mathrm{G})$ and third generation $(3 \mathrm{G})$ networks. The data has been acquired from 75 young and healthy students of a post graduate institute while the students were making their routine calls. The acquired EEG signal is analyzed using various parameters viz.; Approximate Entropy(ApEn), Largest Lyapunov Exponent (LLE), Hurst Exponent (HE), Correlation dimension (CD) and the power of the brain waves have also been analyzed. It has been found that due to mobile phone usage, there is variation in the nonlinear parameters and increase in the power of the alpha brain waves at T5O1 during $3 \mathrm{GRx}$ and decrease in alpha power at the P4O2 channel in all modes. It has been observed that the change at the right temporal region is more, the side to which mobile phone was held. The Statistical analysis has also been done using SPSS software which shows significant variations at some of the channels in different modes.
\end{abstract}

Keywords. Approximate entropy; correlation dimension; electromagnetic radiations; Hurst exponent; largest lyapunov exponent.

\section{Introduction}

The increasing demands for wireless equipments utilizing RF spectrum especially mobile phones have opened up a basis to explore the impact on human beings mainly on the brain. Brain is a vital part of our body and is in close proximity to the radiations from the mobile phones. Radio Frequency (RF) signal is an electromagnetic radiation that has the ability to affect the human body due to its composition of water electrolytes and ions. The exposure due to radiations from towers or other gadgets is on whole body whereas that from the mobile phone handset is a kind of local exposure [1] and is very near to our brain, thus, is a matter of concern. Thermal radiations from the mobile phone is quantified by the specific absorption rate (SAR) value which causes heating of the tissues that increases with the duration of the mobile usage [2]. In view of public and governmental concern, World Health

*For correspondence
Organization established the International Electromagnetic Fields (EMF) in 1996 to assess the adverse effects of these fields. In 2016, WHO also conducted a formal risk assessment study for radiofrequency fields exposures [3]. But in this paper, specifically GSM and WCDMA have been considered which have more users. GSM network is a TDMA based network and operates in the range of 900-1800 MHz. WCDMA operates in the frequency range of 2100-2170 MHz [4]. The possibility of their biological, physiological and psychological effects on the human body has led to the analysis of their effect in detail. Literatures have also pointed to an effect on the EEG by low level RF radiations [5]. The EEG signal beta wave is sensitive to these microwave radiations and depends on its frequency [6].

Electroencephalogram depicts the electrical potential on the scalp of the head acquired using the electrodes. The amplitude of the human EEG is $10-15 \mu \mathrm{V}$ and the frequency is below $30 \mathrm{~Hz}$ [7]. The International 10-20 system is used for the placement of the electrodes. 10 and 20 


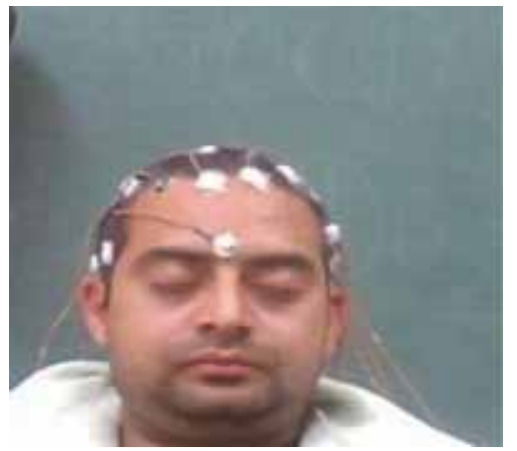

Figure 1. Subject in normal mode.

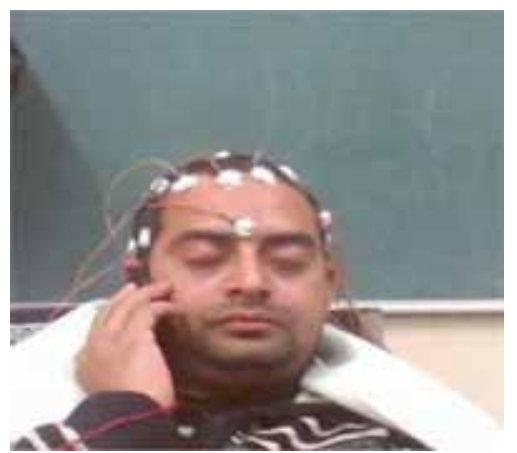

Figure 2. Subject using mobile phone. usually denotes the distance between the electrodes and $10 \%$ or $20 \%$ of the front to back and right to left, placed over the scalp [8]. EEG is due to the neural activation inside the brain that controls the functioning of the whole body system. EEG consists of five brain waves viz; delta $(0.5-4 \mathrm{~Hz})$, theta $(4-8 \mathrm{~Hz})$, alpha $(8-12 \mathrm{~Hz})$, beta1 (12-20 Hz), beta2 (20-30 Hz) [9] and gamma (30-45 Hz). Each wave can be termed as a clock signal for the other body organs for proper functioning and also for the synchronization. Each wave is dominant for a particular activity. In order to analyze the effect of Radio frequency radiations emitted from the mobile phones on these waves, various parameters have been considered. As EEG depicts the non-linear and chaotic behavior hence, non-linear methods also have been utilized to detect the changes in it.

Majority of research work reported in literatures are to know the effect, mainly on the alpha waves. Some researchers do agree that alpha wave get affected due to mobile phone radiations. Others have also reported the effects on the delta waves during the sleep times. Many researchers have also studied the biological effects of mobile phones radiations on students, urban and rural people, etc. Studies have also been done on the animals and found that due to radiations exposure there is an adverse effect on their sleep pattern [10]. The outline of this paper is as follows. Section 1 gives the introduction; section 2 gives the review of researches done on this area whereas section 3 deals with materials and methods. Results and discussions are provided in section 4 and in section 5, conclusions have been presented.

\section{Related study}

Survey was carried out on the available literatures mainly presenting various facts about exposure impact. For the benefit of readers, extract of some of the important references are presented. Roggeveen et al [11] found a

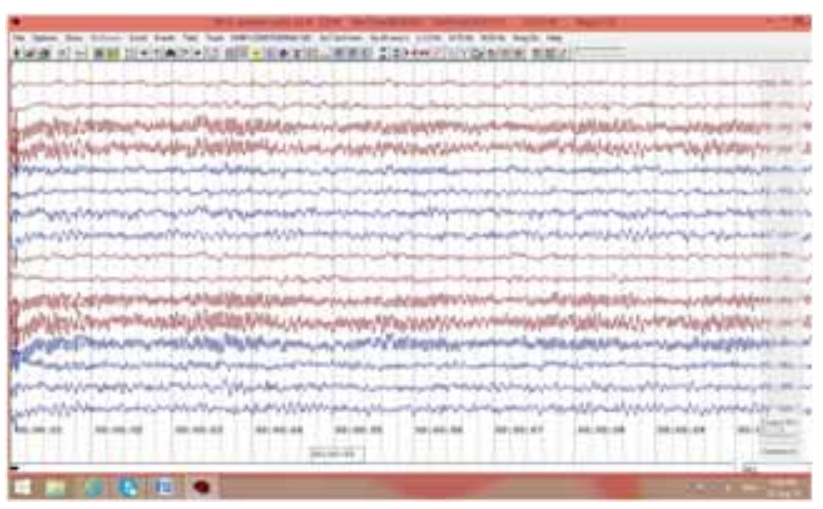

Figure 3. Acquired EEG signal.

significant effect of mobile phone radiations on almost all brain regions and showed increase in alpha, beta, and gamma activity. Reported experimental study found that use of mobile phone on right ear increases more upper beta power leading to muscular discomfort and anxiety [9]. Isa et al [12] experimented on the alpha and beta brain waves showed no decrease in the brain wave power during exposure. Various research studies have also been done on the impact of mobile phone radiations on epileptic subjects which reports on a decrease in the spectral energy of alpha wave at the occipital region and increase in energy for all other brain waves in case of epileptic subjects [13]. Because of the nonlinear nature of the EEG signals, various other algorithms have been employed to measure the nonlinearity, complexity. Hinrikus et al [14] studied the effect of low frequencies microwave radiation on EEG and found significant changes in alpha and beta bands but no effect is seen on theta wave. Smitha et al [15] used fractal dimension algorithm and used mobile phone at different position and analyzed a change in the fractal dimension i.e., change in the complexity of EEG. Nicolas Perentos et al [16] reported decrease in the alpha band due to the mobile phone radiations. Isa et al [17] in their paper concluded that with the radiations from mobile phones 
Table 1. Largest Lyapunov exponent.

\begin{tabular}{|c|c|c|c|c|c|c|c|c|c|}
\hline \multicolumn{6}{|l|}{ Mean } & \multicolumn{4}{|c|}{ p-Value } \\
\hline Channel & Normal & $2 \mathrm{GRx}$ & $2 \mathrm{G} T \mathrm{x}$ & $3 \mathrm{G} \mathrm{Rx}$ & 3G Tx & $2 \mathrm{G} R \mathrm{x}$ & $2 \mathrm{G} T \mathrm{x}$ & $3 \mathrm{G} \mathrm{Rx}$ & $3 \mathrm{G} \mathrm{Tx}$ \\
\hline C3P3 & 0.009708 & 0.012961 & 0.017508 & 0.012473 & 0.014182 & 0.33 & 0.07 & 0.52 & 0.308 \\
\hline C4P4 & 0.011025 & 0.01555 & 0.019521 & 0.013892 & 0.013766 & 0.33 & 0.07 & 0.52 & 0.308 \\
\hline $\mathrm{F} 3 \mathrm{C} 3$ & 0.004789 & 0.005585 & 0.004786 & 0.006177 & 0.007231 & 0.682 & 0.999 & 0.651 & 0.281 \\
\hline $\mathrm{F} 4 \mathrm{C} 4$ & 0.011512 & 0.008222 & 0.007392 & 0.009135 & 0.008797 & 0.315 & 0.296 & 0.504 & 0.364 \\
\hline F7T3 & 0.012158 & 0.009238 & 0.016246 & 0.01197 & 0.007485 & 0.65 & 0.643 & 0.969 & 0.409 \\
\hline F8T4 & 0.012353 & 0.010554 & 0.014635 & 0.017404 & 0.017546 & 0.607 & 0.659 & 0.438 & 0.637 \\
\hline FP1F3 & 0.013569 & 0.005501 & 0.017235 & 0.010964 & 0.00796 & 0.133 & 0.605 & 0.672 & 0.201 \\
\hline FP1F7 & 0.01368 & 0.008319 & 0.020042 & 0.016676 & 0.00998 & 0.268 & 0.478 & 0.692 & 0.39 \\
\hline FP2F4 & 0.011462 & 0.004678 & 0.012292 & 0.011491 & 0.005286 & 0.055 & 0.892 & 0.996 & 0.129 \\
\hline FP2F8 & 0.012976 & 0.010615 & 0.015177 & 0.014985 & 0.007444 & 0.589 & 0.715 & 0.71 & 0.12 \\
\hline P3O1 & 0.017188 & 0.015481 & 0.022327 & 0.019256 & 0.019953 & 0.692 & 0.546 & 0.539 & 0.658 \\
\hline $\mathrm{P} 4 \mathrm{O} 2$ & 0.019896 & 0.032523 & 0.016155 & 0.013417 & 0.012809 & 0.541 & 0.701 & 0.252 & 0.332 \\
\hline T3T5 & 0.014581 & 0.009754 & 0.01663 & 0.015053 & 0.012223 & 0.378 & 0.778 & 0.899 & 0.661 \\
\hline T4T6 & 0.010817 & 0.01265 & 0.021046 & 0.013573 & 0.013073 & 0.602 & 0.14 & 0.594 & 0.599 \\
\hline T5O1 & 0.01255 & 0.007704 & 0.018127 & 0.010937 & 0.016046 & 0.085 & 0.491 & 0.672 & 0.384 \\
\hline T6O2 & 0.018908 & 0.02686 & 0.018323 & 0.00871 & 0.010877 & 0.67 & 0.947 & 0.31 & 0.441 \\
\hline
\end{tabular}

Table 2. Approximate entropy.

\begin{tabular}{|c|c|c|c|c|c|c|c|c|c|}
\hline \multicolumn{6}{|l|}{ Mean } & \multicolumn{4}{|c|}{ p-Value } \\
\hline Channel & Normal & $2 \mathrm{G} R \mathrm{x}$ & $2 \mathrm{G} T \mathrm{x}$ & 3G Rx & 3G Tx & $2 \mathrm{G} R \mathrm{x}$ & $2 \mathrm{G} T \mathrm{x}$ & 3G Rx & $3 \mathrm{G} T \mathrm{x}$ \\
\hline C3P3 & 0.619731 & 0.635046 & 0.62055 & 0.638077 & 0.626812 & 0.316 & 0.968 & 0.406 & 0.703 \\
\hline $\mathrm{C} 4 \mathrm{P} 4$ & 0.6277 & 0.626369 & 0.608665 & 0.632023 & 0.633527 & 0.903 & 0.435 & 0.824 & 0.767 \\
\hline F3C3 & 0.609308 & 0.620915 & 0.635512 & 0.629146 & 0.618954 & 0.597 & 0.352 & 0.53 & 0.735 \\
\hline $\mathrm{F} 4 \mathrm{C} 4$ & 0.621608 & 0.606773 & 0.582973 & 0.628208 & 0.619073 & 0.521 & 0.135 & 0.815 & 0.927 \\
\hline F7T3 & 0.590858 & 0.577415 & 0.580596 & 0.607385 & 0.613004 & 0.716 & 0.651 & 0.587 & 0.375 \\
\hline F8T4 & 0.616069 & 0.622573 & 0.604108 & 0.630585 & 0.639565 & 0.865 & 0.571 & 0.74 & 0.432 \\
\hline FP1F3 & 0.491292 & 0.496685 & 0.520873 & 0.5189 & 0.520619 & 0.893 & 0.36 & 0.538 & 0.416 \\
\hline FP1F7 & 0.506208 & 0.495815 & 0.532458 & 0.514808 & 0.536712 & 0.817 & 0.412 & 0.83 & 0.31 \\
\hline FP2F4 & 0.565492 & 0.554827 & 0.551054 & 0.545812 & 0.563673 & 0.797 & 0.71 & 0.657 & 0.965 \\
\hline FP2F8 & 0.574488 & 0.566915 & 0.562427 & 0.56425 & 0.574115 & 0.827 & 0.691 & 0.79 & 0.989 \\
\hline P3O1 & 0.626381 & 0.649881 & 0.608312 & 0.644481 & 0.654665 & 0.12 & 0.434 & 0.492 & 0.177 \\
\hline $\mathrm{P} 4 \mathrm{O} 2$ & 0.643304 & 0.641854 & 0.617804 & 0.637908 & 0.632669 & 0.923 & 0.218 & 0.847 & 0.571 \\
\hline T3T5 & 0.609773 & 0.632669 & 0.602327 & 0.636696 & 0.631935 & 0.25 & 0.586 & 0.262 & 0.249 \\
\hline T4T6 & 0.606796 & 0.634212 & 0.596238 & 0.623731 & 0.635938 & 0.193 & 0.637 & 0.557 & 0.229 \\
\hline T5O1 & 0.617642 & 0.642404 & 0.612692 & 0.655792 & 0.677962 & 0.172 & 0.811 & 0.239 & $0.024 *$ \\
\hline T6O2 & 0.648808 & 0.670827 & 0.635412 & 0.661381 & 0.653673 & 0.297 & 0.629 & 0.486 & 0.801 \\
\hline
\end{tabular}

*Significant values.

there is an imbalance in both left and right brains. Murat [18] have also studied the alpha brain waves and found significant decrease in the alpha waves. Some studies also showed that continuous or pulsed radio frequency exposures do not cause change in the alpha brain wave [5]. The experiment performed separately on both sides of brain showed reduced symmetry between parts of the brain and reduced correlation between beta and alpha waves after the mobile phone radiation exposure [19]. Luis et al [20] studied the effects of cell phone radiation on the EEG spectra of epileptic patients and found that there was an increase in beta and gamma power compared to a normal non-exposed persons. Thermographic analysis has also been done by Vorst et al [21]. They reported an increase in the temperature at the surface of ear and nearby in the range 1.0 to $2.4 \pm 0.2^{\circ} \mathrm{C}$. Similarly Piuzzi et al [22] did an analysis of effect of radiations at mobile communication frequencies on child and adults models on the basis of on the Specific Absorption Rate (SAR) value and reported that the level specified increases in case of child. Lin [23] studied that those students who utilize mobile phone more in chatting and doing SMS show less pick up, delayed and 


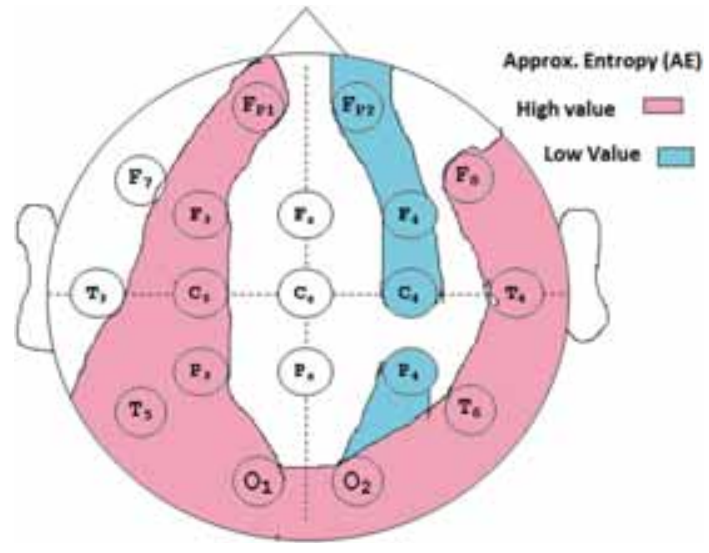

Figure 4. Approximate entropy and Channel-wise impact.

inaccurate responses [23]. Ilvonen et al [24] analyzed the effect of magnetic field induced through GSM mobile phones was considerably lower than the basic restriction limit given. The biological effects of mobile phone radiations studied in literature includes anxiety, sleep disorders, memory loss, pain in ear and temperature rise [25] in surrounding nerves, complicated birth and miscarriages in case of pregnant woman, effect on eyes [26]. In contrast to the research on increase in the alpha band power, in 2007 Perentos et al [27] found no significant change in the alpha power due to mobile phone radiations. Lin [28] has done various experiments on the sleep activity changes by exposure to the GSM mobile phone radiations. The author found inconsistent results in the sleep behavior. Researches have also been done on the micro structure of sleep and reported no effect due to GSM and WCDMA mobile phones [29]. The research has also resulted in favor of these radiations, by showing better correlation between delta and theta brain waves after exposure and hence improved brain synchronization [30]. The GSM effect on brain is high as the power spectral density (PSD) of EEG signal is more when exposed to GSM mobile phone as compared to CDMA network phone [31]. The mixed reports motivated the authors to have a close look into the effect on EEG by conducting experiments in users' realistic conditions.

\section{Materials and methods}

\subsection{Subjects}

This study has been conducted on 75 (57 males and 18 females) young and healthy students studying in post graduate courses at National Institute of Technical Teachers Training and Research, Chandigarh, India. The mean age of the subjects is 22.2 years with standard deviation of 2.27 years. The subjects' daily usage of mobile phones is between $2-3 \mathrm{~h}$. The subjects are healthy and not on any kind of medicine. While recordings are done, these subjects used their $2 \mathrm{G}$ and 3G mobile phones. In the experiments, Nokia, Samsung, Panasonic and Motorola mobile phones having SAR value between 0.67 and $1.14 \mathrm{~W} / \mathrm{kg}$ are used by the subjects. The recordings are done when the subjects made their routine calls. So no external or any kind of $\mathrm{RF}$ exposure is given. Informed consent has been obtained individually from all the participants included in the study.

Table 3. Correlation dimension.

\begin{tabular}{|c|c|c|c|c|c|c|c|c|c|}
\hline \multicolumn{6}{|l|}{ Mean } & \multicolumn{4}{|c|}{ p-Value } \\
\hline Channel & Normal & 2G Rx & 2G Tx & 3G Rx & 3G Tx & $2 \mathrm{G} R \mathrm{x}$ & 2G Tx & 3G Rx & $3 \mathrm{G} T \mathrm{x}$ \\
\hline C3P3 & 2.081912 & 2.123804 & 2.079019 & 2.085765 & 2.065169 & 0.494 & 0.893 & 0.898 & 0.507 \\
\hline C4P4 & 2.117642 & 2.121315 & 2.12835 & 2.113377 & 2.100565 & 0.838 & 0.587 & 0.874 & 0.481 \\
\hline $\mathrm{F} 3 \mathrm{C} 3$ & 1.850623 & 1.908758 & 1.836592 & 1.856327 & 1.851708 & 0.443 & 0.502 & 0.866 & 0.972 \\
\hline F4C4 & 1.907273 & 1.897954 & 1.850465 & 1.896838 & 1.879673 & 0.787 & 0.109 & 0.834 & 0.55 \\
\hline F7T3 & 1.914812 & 2.006081 & 1.848527 & 1.894096 & 1.849254 & 0.329 & 0.11 & 0.62 & 0.135 \\
\hline F8T4 & 1.95965 & 1.965142 & 1.950662 & 1.963335 & 1.907988 & 0.858 & 0.778 & 0.93 & 0.176 \\
\hline FP1F3 & 1.821912 & 1.905673 & 1.793927 & 1.850208 & 1.815442 & 0.2 & 0.43 & 0.612 & 0.893 \\
\hline FP1F7 & 1.898312 & 1.965888 & 1.846265 & 1.909642 & 1.862119 & 0.296 & 0.148 & 0.833 & 0.4 \\
\hline FP2F4 & 1.808996 & 1.852881 & 1.782369 & 1.809427 & 1.796762 & 0.609 & 0.472 & 0.994 & 0.778 \\
\hline FP2F8 & 1.914862 & 1.942169 & 1.917223 & 1.944631 & 1.900677 & 0.327 & 0.951 & 0.601 & 0.754 \\
\hline P3O1 & 2.077715 & 2.2027 & 2.110981 & 2.104835 & 2.110973 & 0.123 & 0.255 & 0.405 & 0.405 \\
\hline $\mathrm{P} 4 \mathrm{O} 2$ & 2.099054 & 2.090773 & 2.08345 & 2.089773 & 2.043246 & 0.714 & 0.609 & 0.826 & 0.11 \\
\hline T3T5 & 2.030035 & 2.116596 & 2.034423 & 2.042296 & 2.015485 & 0.237 & 0.906 & 0.698 & 0.647 \\
\hline T4T6 & 2.025527 & 2.108208 & 2.033419 & 2.029796 & 2.017158 & 0.285 & 0.774 & 0.913 & 0.784 \\
\hline T5O1 & 1.929312 & 2.0555 & 1.971462 & 1.969208 & 1.972819 & 0.103 & 0.08 & 0.195 & 0.264 \\
\hline T6O2 & 1.987658 & 2.041146 & 2.000962 & 1.978512 & 1.950792 & 0.384 & 0.691 & 0.804 & 0.236 \\
\hline
\end{tabular}


Table 4. Hurst exponent.

\begin{tabular}{|c|c|c|c|c|c|c|c|c|c|}
\hline \multicolumn{6}{|l|}{ Mean } & \multicolumn{4}{|c|}{ p-Value } \\
\hline Channel & Normal & 2G Rx & $2 \mathrm{G} T \mathrm{x}$ & 3G Rx & 3G Tx & $2 \mathrm{G} \mathrm{Rx}$ & $2 \mathrm{G} T \mathrm{x}$ & 3G Rx & $3 G \mathrm{Tx}$ \\
\hline C3P3 & 0.67078 & 0.71531 & 0.69472 & 0.67788 & 0.68517 & 0.131 & 0.33 & 0.842 & 0.669 \\
\hline $\mathrm{C} 4 \mathrm{P} 4$ & 0.66278 & 0.660177 & 0.706404 & 0.698258 & 0.665215 & 0.92 & 0.149 & 0.31 & 0.927 \\
\hline F3C3 & 0.77421 & 0.783369 & 0.736092 & 0.699165 & 0.76941 & 0.77 & 0.159 & 0.064 & 0.885 \\
\hline $\mathrm{F} 4 \mathrm{C} 4$ & 0.69396 & 0.745392 & 0.756815 & 0.768335 & 0.7517 & 0.089 & $0.014^{*}$ & $0.014 *$ & $0.02 *$ \\
\hline F7T3 & 0.73179 & 0.825796 & 0.783092 & 0.765958 & 0.781077 & $0.018 *$ & $0.031 *$ & 0.296 & 0.13 \\
\hline F8T4 & 0.73331 & 0.75355 & 0.771096 & 0.758869 & 0.738085 & 0.499 & 0.168 & 0.427 & 0.828 \\
\hline FP1F3 & 0.78238 & 0.825581 & 0.804754 & 0.7755 & 0.766592 & 0.131 & 0.364 & 0.801 & 0.571 \\
\hline FP1F7 & 0.77450 & 0.807381 & 0.778738 & 0.775992 & 0.772904 & 0.273 & 0.889 & 0.961 & 0.957 \\
\hline $\mathrm{FP} 2 \mathrm{~F} 4$ & 0.79990 & 0.787127 & 0.781554 & 0.781042 & 0.773027 & 0.507 & 0.451 & 0.431 & 0.239 \\
\hline FP2F8 & 0.76183 & 0.793592 & 0.786273 & 0.749473 & 0.765573 & 0.279 & 0.434 & 0.627 & 0.896 \\
\hline P3O1 & 0.75805 & 0.737277 & 0.698181 & 0.732008 & 0.695985 & 0.418 & $0.033^{*}$ & 0.349 & 0.096 \\
\hline $\mathrm{P} 4 \mathrm{O} 2$ & 0.69652 & 0.699262 & 0.718419 & 0.702231 & 0.708735 & 0.938 & 0.429 & 0.843 & 0.676 \\
\hline T3T5 & 0.74845 & 0.761519 & 0.747931 & 0.769342 & 0.758446 & 0.691 & 0.985 & 0.431 & 0.729 \\
\hline T4T6 & 0.70300 & 0.734008 & 0.703788 & 0.720196 & 0.747104 & 0.396 & 0.974 & 0.626 & 0.221 \\
\hline T5O1 & 0.81837 & 0.764973 & 0.755242 & 0.786258 & 0.744262 & $0.038 *$ & $0.025^{*}$ & $0.369 *$ & $0.013^{*}$ \\
\hline T6O2 & 0.71648 & 0.784142 & 0.775635 & 0.716673 & 0.730512 & 0.065 & $0.038^{*}$ & 0.994 & 0.635 \\
\hline
\end{tabular}

*Significant values.

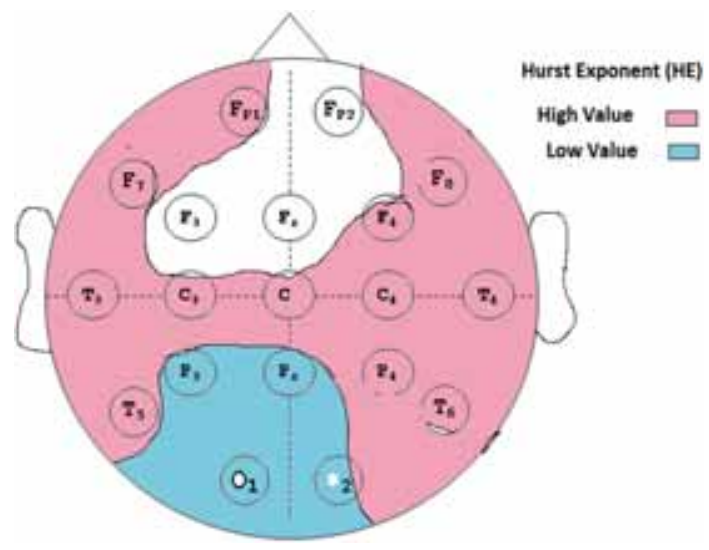

Figure 5. Hurst Exponent Channel-wise impact.

\subsection{Protocol}

The EEG acquisition is done in the morning before 8 a.m. and classified as: State when no mobile communication is going on i.e., the subject is in a relax mode, the EEG signal so acquired is called normal or ideal mode signal. Other states are when the subjects used mobile phones in GSM network (2G) and WCDMA network (3G) in transmitting and receiving modes. Tx-when the call is initiated by the subject and is called transmission mode, $\mathrm{Rx}$ - when the call is received. The sequence of recording is not fixed and is random as the state comes. The experiment is done in a laboratory where there is no localized generation of EM signals except the signal available from the mobile towers responsible for establishing usual mobile communications. The same is ensured by using a Boonton Power meter Model no. 52018 having a sensitivity of nano watts. The EEG signal of the subject is recorded without a mobile phone and the baseline is defined for the concerned subject based on the EEG recorded in the absence of the use of mobile phone. The subject is then exposed to EM radiations by allowing use of mobile phone both in transmission as well as receiving mode. The recorded EEG signal is compared with the baseline signal as stated above and the variations so obtained are analyzed. As a pre-filtering process to ensure that the cable of the EEG nodes are not picking up any external signal from the mobile, the experiment is performed by putting the mobile on a phantom model and fixing the sensors on the phantom head to ensure that proper shielding of the EEG sensor node and wires exist. The set-up with dummy and the subsequent recorded EEG with dummy is a preprocessing method carried out to eliminate the noise arises due to picking of the signal by the EEG wires externally from the mobile phones. The field intensity is measured using a Boonton power meter Model no. 52018 before the acquisition is done. No appreciable field intensity variation is observed till $10 \mathrm{~min}$. Hence, the measurement is carried out up to 5 min therefore, is in a non-varying situation. EMI shielding is an essential experimental need to ensure accurate study in this type of research where the signal level is quite low and is distributed over various bands of frequency. In this experiment, 16 channel EEG recorder is used. In order to shield the external signal, each channel is connected to phantom separately i.e., when channel C3P3 is connected, 
Table 5. Delta power.

\begin{tabular}{|c|c|c|c|c|c|c|c|c|c|}
\hline \multicolumn{6}{|l|}{ Mean } & \multicolumn{4}{|c|}{ p-Value } \\
\hline Channel & Normal & $2 \mathrm{GRx}$ & $2 \mathrm{G} T \mathrm{x}$ & 3G Rx & 3G Tx & $2 \mathrm{G} \mathrm{Rx}$ & $2 \mathrm{G} T \mathrm{x}$ & 3G Rx & 3G Tx \\
\hline C3P3 & 70.04701 & 81.15355 & 74.89917 & 66.71412 & 54.21314 & 0.635 & 0.801 & 0.872 & 0.388 \\
\hline $\mathrm{C} 4 \mathrm{P} 4$ & 52.48661 & 61.1039 & 64.00522 & 70.37273 & 53.31393 & 0.702 & 0.498 & 0.508 & 0.955 \\
\hline F3C3 & 66.99561 & 40.59951 & 48.45803 & 69.06251 & 79.31853 & 0.304 & 0.416 & 0.963 & 0.837 \\
\hline $\mathrm{F} 4 \mathrm{C} 4$ & 89.54494 & 69.46851 & 63.76374 & 393.4787 & 59.29394 & 0.598 & 0.472 & 0.353 & 0.489 \\
\hline F7T3 & 121.3372 & 194.2645 & 77.24941 & 95.39073 & 155.5642 & 0.375 & 0.114 & 0.437 & 0.708 \\
\hline F8T4 & 67.16519 & 129.1135 & 63.60401 & 124.1656 & 75.60461 & 0.103 & 0.711 & 0.177 & 0.706 \\
\hline FP1F3 & 178.6189 & 195.9787 & 143.2456 & 285.8682 & 158.2611 & 0.814 & 0.414 & 0.303 & 0.749 \\
\hline FP1F7 & 192.5157 & 349.0423 & 157.4349 & 202.0418 & 201.1452 & 0.298 & 0.363 & 0.829 & 0.887 \\
\hline FP2F4 & 129.3849 & 138.93 & 109.9932 & 456.1884 & 154.3886 & 0.809 & 0.337 & 0.308 & 0.444 \\
\hline FP2F8 & 137.2698 & 206.1465 & 134.7982 & 132.1314 & 193.7105 & 0.419 & 0.907 & 0.853 & 0.325 \\
\hline P3O1 & 105.0073 & 119.2044 & 121.0279 & 82.53108 & 75.44238 & 0.598 & 0.565 & 0.436 & 0.232 \\
\hline $\mathrm{P} 4 \mathrm{O} 2$ & 267.4399 & 110.1149 & 95.13507 & 109.7379 & 81.46641 & 0.288 & 0.31 & 0.383 & 0.281 \\
\hline T3T5 & 99.3516 & 99.66959 & 91.22184 & 89.3623 & 84.98933 & 0.992 & 0.572 & 0.484 & 0.623 \\
\hline T4T6 & 106.8207 & 99.91615 & 74.32863 & 62.82903 & 92.15032 & 0.74 & 0.207 & 0.216 & 0.741 \\
\hline T5O1 & 105.1323 & 160.4738 & 127.6208 & 111.2924 & 97.61465 & 0.479 & 0.655 & 0.807 & 0.826 \\
\hline T6O2 & 231.4494 & 96.2922 & 66.33618 & 69.06804 & 65.35798 & 0.368 & 0.331 & 0.315 & 0.294 \\
\hline
\end{tabular}

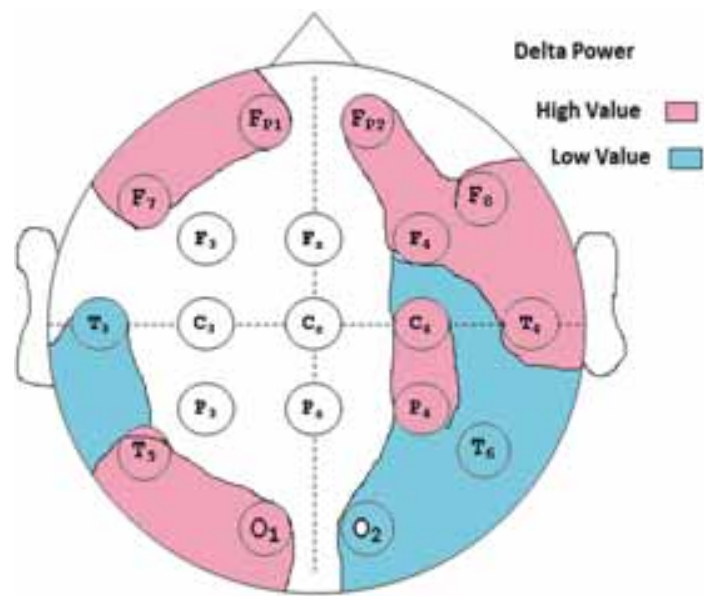

Figure 6. Delta power channel-wise impact.

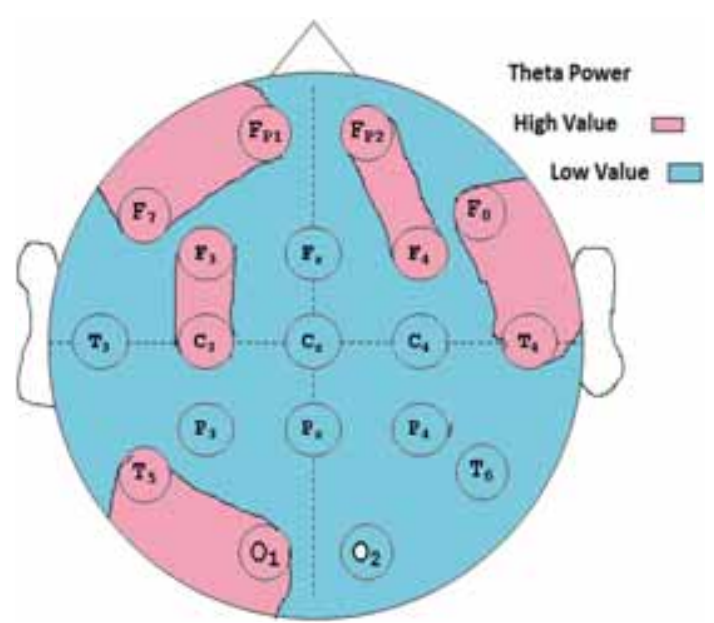

Figure 7. Theta Power Channel-wise impact. no other sensor node is connected and so on for all the other 16 channels. After each channel is tested separately, the channels are connected sequentially and are verified for any external signal. Both approaches i.e., measuring the absolute EMI for each channel and average measured over duration of 10 min yield no EMI due to connecting wire or sensor nodes. The nano-watt power meter shows no sign of any signal being picked up by the sensor node/ connecting wire of EEG. The same was shown to the Senior Cardiologist. Hence, the variations observed in brain waves when mobile phone is used by subjects are due to RF radiation absorbed in the head but not by any external signal through connecting wires/sensor node.

\subsection{Data acquisition}

EEG signals are acquired by placing 20 electrodes on the scalp in 10-20 international system configuration using RMS Maximus 24-32 set-up. The F, T, P, C and O stands for the part of the scalp as frontal, temporal, Parietal, central and Occipital, respectively [8]. Even numbers 2, 4, 6 , etc. refer to the right position and odd numbers $1,3,5$, etc. refer to the left positioning of electrodes on the scalp of the brain [8]. Electrode impedances are below $5 \mathrm{~K}$ ohm at the start of the recordings. As shown in figure 1, the subject is in normal mode i.e., recording is done without mobile phone and in figure 2 the subject is in communicating 
Table 6. Theta power.

\begin{tabular}{|c|c|c|c|c|c|c|c|c|c|}
\hline \multicolumn{6}{|l|}{ Mean } & \multicolumn{4}{|c|}{ p-Value } \\
\hline Channel & Normal & $2 \mathrm{GRx}$ & $2 \mathrm{G} T \mathrm{x}$ & 3G Rx & 3G Tx & $2 \mathrm{G} \mathrm{Rx}$ & $2 \mathrm{G} T \mathrm{x}$ & 3G Rx & 3G Tx \\
\hline C3P3 & 18.31366 & 15.49625 & 21.96054 & 16.02575 & 16.17433 & 0.125 & 0.325 & 0.384 & 0.407 \\
\hline $\mathrm{C} 4 \mathrm{P} 4$ & 16.78444 & 15.42868 & 16.26767 & 16.53605 & 16.85979 & 0.152 & 0.452 & 0.822 & 0.948 \\
\hline F3C3 & 6.856492 & 6.739665 & 7.125281 & 7.651981 & 9.222648 & 0.743 & 0.411 & 0.444 & 0.297 \\
\hline $\mathrm{F} 4 \mathrm{C} 4$ & 8.878698 & 7.272535 & 7.403224 & 25.5249 & 8.379102 & 0.237 & 0.391 & 0.336 & 0.791 \\
\hline F7T3 & 24.1441 & 12.9009 & 6.856674 & 7.795781 & 11.21104 & 0.339 & 0.321 & 0.353 & 0.483 \\
\hline F8T4 & 7.267655 & 7.52023 & 6.963392 & 8.341928 & 8.509558 & 0.794 & 0.775 & 0.551 & 0.444 \\
\hline FP1F3 & 8.002909 & 7.538124 & 8.457346 & 9.398506 & 7.622516 & 0.735 & 0.591 & 0.641 & 0.813 \\
\hline FP1F7 & 8.370889 & 8.321811 & 9.463854 & 10.04721 & 10.24994 & 0.97 & 0.157 & 0.588 & 0.421 \\
\hline FP2F4 & 6.600333 & 5.767204 & 6.854813 & 35.7491 & 6.97565 & 0.367 & 0.773 & 0.321 & 0.748 \\
\hline FP2F8 & 10.82959 & 9.505129 & 10.15997 & 9.648896 & 9.883322 & 0.553 & 0.765 & 0.721 & 0.768 \\
\hline P3O1 & 19.83688 & 16.3249 & 21.79547 & 19.19764 & 17.11702 & $0.023^{*}$ & 0.672 & 0.852 & 0.276 \\
\hline $\mathrm{P} 4 \mathrm{O} 2$ & 30.0366 & 13.95981 & 12.90312 & 13.84544 & 13.6298 & 0.217 & 0.204 & 0.229 & 0.224 \\
\hline T3T5 & 19.3111 & 13.57945 & 11.8235 & 12.06555 & 11.22318 & 0.31 & 0.362 & 0.374 & 0.334 \\
\hline T4T6 & 11.25739 & 9.170726 & 9.525009 & 9.883831 & 11.66336 & 0.123 & 0.234 & 0.373 & 0.852 \\
\hline T5O1 & 10.0458 & 10.08923 & 9.621125 & 12.74459 & 11.40402 & 0.942 & 0.521 & 0.263 & 0.119 \\
\hline T6O2 & 32.3783 & 9.858688 & 9.897941 & 9.260502 & 9.055266 & 0.321 & 0.322 & 0.308 & 0.303 \\
\hline
\end{tabular}

Table 7. Alpha power.

\begin{tabular}{|c|c|c|c|c|c|c|c|c|c|}
\hline \multicolumn{6}{|l|}{ Mean } & \multicolumn{4}{|c|}{ p-Value } \\
\hline Channel & Normal & $2 \mathrm{G} R \mathrm{x}$ & $2 \mathrm{G} T \mathrm{x}$ & 3G Rx & 3G Tx & $2 \mathrm{G} \mathrm{Rx}$ & 2G Tx & 3G Rx & $3 \mathrm{G} T \mathrm{x}$ \\
\hline $\mathrm{C} 3 \mathrm{P} 3$ & 111.6407 & 114.8864 & 125.6605 & 129.7871 & 121.4189 & 0.511 & $0.031 *$ & $0.042 *$ & 0.098 \\
\hline C4P4 & 94.67723 & 104.7858 & 109.8014 & 113.0054 & 109.9707 & 0.081 & $0.015^{*}$ & $0.033^{*}$ & $0.038 *$ \\
\hline $\mathrm{F} 3 \mathrm{C} 3$ & 21.43226 & 21.41355 & 21.8796 & 23.87838 & 24.14033 & 0.99 & 0.636 & 0.3 & 0.356 \\
\hline $\mathrm{F} 4 \mathrm{C} 4$ & 27.55644 & 27.20852 & 27.65807 & 114.2335 & 31.0259 & 0.903 & 0.968 & 0.306 & 0.335 \\
\hline F7T3 & 34.32952 & 23.99076 & 17.68259 & 20.06277 & 20.14169 & 0.369 & 0.355 & 0.431 & 0.442 \\
\hline F8T4 & 22.1681 & 24.11161 & 24.64295 & 26.78337 & 26.34563 & 0.217 & 0.056 & 0.077 & 0.057 \\
\hline FP1F3 & 6.956062 & 6.478942 & 6.503286 & 8.326383 & 6.793317 & 0.612 & 0.704 & 0.575 & 0.915 \\
\hline FP1F7 & 10.07723 & 10.16053 & 10.2453 & 12.07927 & 11.80021 & 0.919 & 0.88 & 0.409 & 0.292 \\
\hline FP2F4 & 6.232504 & 5.475342 & 5.977054 & 119.0231 & 5.785321 & 0.448 & 0.786 & 0.152 & 0.686 \\
\hline FP2F8 & 15.01684 & 13.9011 & 13.89094 & 14.90652 & 14.32487 & 0.688 & 0.751 & 0.976 & 0.855 \\
\hline P3O1 & 67.90402 & 63.44351 & 73.91536 & 68.44906 & 68.68106 & 0.1 & 0.328 & 0.87 & 0.852 \\
\hline $\mathrm{P} 4 \mathrm{O} 2$ & 92.77627 & 56.00431 & 59.92079 & 60.18076 & 57.75913 & $0.003 *$ & $0.007 *$ & $0.01 *$ & $0.004 *$ \\
\hline T3T5 & 51.35655 & 49.09019 & 47.13861 & 48.50372 & 47.13089 & 0.715 & 0.562 & 0.715 & 0.593 \\
\hline T4T6 & 51.40668 & 50.39165 & 52.83726 & 54.71222 & 54.10506 & 0.123 & 0.234 & 0.373 & 0.852 \\
\hline T5O1 & 21.14848 & 24.88421 & 24.40096 & 29.4612 & 26.58303 & 0.091 & 0.074 & $0.045^{*}$ & 0.071 \\
\hline T6O2 & 57.32906 & 24.17881 & 23.74197 & 25.93086 & 25.12119 & 0.352 & 0.347 & 0.381 & 0.368 \\
\hline
\end{tabular}

*Significant values.

mode. The subjects are asked to close the eyes to avoid noises due to eye blinking.

The EEG signal acquired is shown in figure 3. After acquisition, the signal is filtered through $1 \mathrm{~Hz}$ filter; $50 \mathrm{~Hz}$ filter and EMG filter for the various noises. The sampling frequency is $256 \mathrm{~Hz}$. The signal is then taken into excel format and processed for various parameters using programs code written in MATLAB. For calculating the power, the EEG is first extracted into all brain waves viz. delta, theta, alpha, beta and gamma using IIR Butterworth band pass filter with lower and upper cut-off frequency at the specified range of each brain wave.

\subsection{Methodology}

The EEG raw data so recorded from all the five modes is analyzed for various linear and nonlinear parameters 
as follows. The data after filtering is first extracted into excel format and then values for each channel for each parameter is calculated using the MATLAB codes. The analysis is done for Largest Lyapunov Exponent (LLE), Hurst Exponent, Approximate Entropy (ApEn), Correlation Dimension (CD) and Power for all the five brain waves. LLE is a parameter used to measure the chaos in the system or time signal and is a measure of stability. It is an average value of long data time series and denotes the overall characteristics of the data. It can be positive, negative and zero depicting the various states of the data. The system is stable if LLE is less than zero. When it is positive, it represents a chaotic condition and at boundary when equals to zero [32]. LLE is mostly used to find the non-linearity in the system. Approximate entropy (ApEn) gives the amount of irregularity in the signal. A high value of ApEn denotes higher irregularity and lower value denotes the regularity in the patterns of the signal [33]. The embedding dimension ' $\mathrm{m}$ ' in the algorithm of ApEn is the length of the segments for the input series that are compared and ' $r$ ' is the threshold that is fixed for the comparison of the segments [34]. The ApEn value is calculated in the steps as explained in [35]. High value of correlation dimension (CD) denotes the signal is more complex and less similarity in the data [36]. Hurst Exponent (HE) tells about the smoothness of the time series data and is dependent on the past samples [37]. If the value is close to zero, it means smoothness in the data is less [37]. The EEG signal is also extracted into the five brain waves namely delta, theta, alpha, beta and gamma by using band pass filter for each channel and for each subject. Their mean power is calculated. The mean values of each parameter is calculated and compared with the ideal value for each channel. The results so obtained are then statistically analyzed using SPSS software [38] version 20. The mean values are compared in 2-tailed t-test and the p-value [39] i.e., probability value, below 0.05 is taken as significant which means that the actual change in the statistics is significant and is to be considered and the null hypotheses is rejected. In this research work, the null hypothesis is that there is no significant difference in the mean of the two variables.

\section{Results and discussion}

The mean values of all parameters are taken and the results so obtained are depicted in tables 1 to 9 as shown. The mean values for all modes i.e., normal, 2GRx, 2GTx, 3GRx, and 3GTx have been presented and corresponding to each mode their ' $p$ ' value from the statistical analysis is shown. The values of Lyapunov exponent depicted in table 1 shows the behavior of the system. The positive Lyapunov exponent depicts the chaotic situation and the negative exponent depicts the regular situation [36]. The higher value of LLE depicts more chaotic behavior. In 2GRx, LLE value is higher in 2GTx mode mostly at all channels and low in 2GRx mode. The highest value is at temporal, occipital and all parietal sections in the brain during $2 \mathrm{G}$ transmission mode of communication. But the value is not significantly high at any of the channel. Approximate entropy as depicted in table 2, increases with the use of mobile phone but not significant to make a claim except during 3GTx at T5O1 channel. The increase in approximate entropy shows that the complexity of the signal increases with the exposure. There is higher change in the FP2F4 and F4C4, as shown in figure 4 which is near to the right ear i.e., the side on which mobile phone was held.

This supports the findings of Ilvonen et al [24] that current drops rapidly when moving away from the source, so the effect is near to the source. It has been

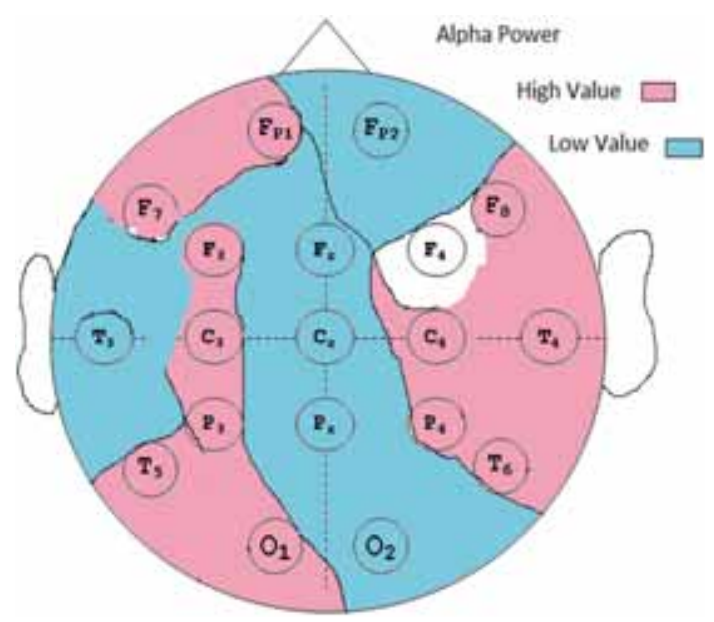

Figure 8. Alpha power channel-wise impact.

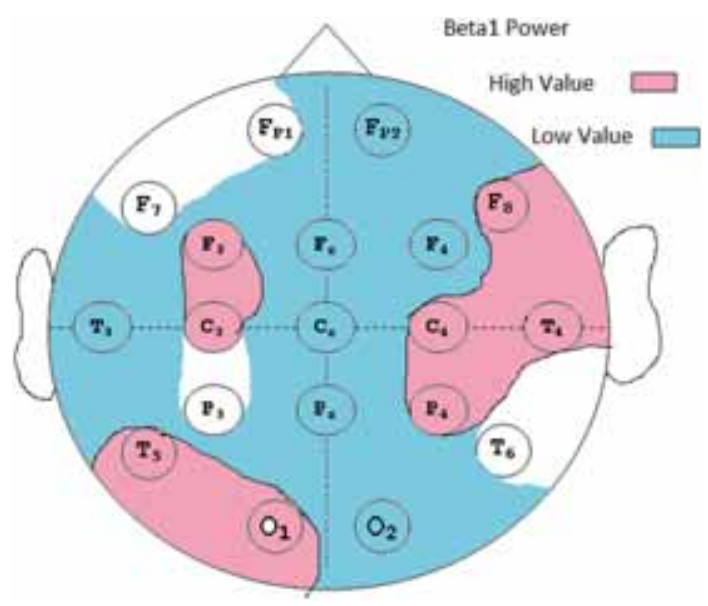

Figure 9. Beta power channel-wise impact. 
found in [40] that EEG of Alzheimer's patient is less complex and the value of approximate entropy in them is significantly lower than the normal control group. Correlation dimension parameter defines the dimension of the signal and with the exposure there is no significant change in the dimension parameter. It is approximately same throughout as shown in table 3. Hurst Exponent as given in table 4 is significantly high at $\mathrm{F} 4 \mathrm{C} 4$ during all the modes. At some channels its trend is on higher while on in other modes it is on the lower side. The value at
T5O1 is significantly low in all the modes. As shown in figure 5, the value is low at the occipital region and there is no change in the frontal part of the brain whereas it is high at the central and temporal part.

The delta wave prevails during the deep sleep. With the use of mobile phones, there is no significant change observed as depicted in table 5. When analyzing the individual channels on the brain in figure 6 , it is observed that the values are higher at the right temporal

Table 8. Beta power.

\begin{tabular}{|c|c|c|c|c|c|c|c|c|c|}
\hline \multicolumn{6}{|l|}{ Mean } & \multicolumn{4}{|c|}{ p-Value } \\
\hline Channel & Normal & $2 \mathrm{G} R \mathrm{x}$ & $2 \mathrm{G} T \mathrm{x}$ & $3 \mathrm{G} \mathrm{Rx}$ & $3 \mathrm{G} T \mathrm{x}$ & $2 \mathrm{G} T \mathrm{x}$ & $2 \mathrm{G} T \mathrm{x}$ & 3G Rx & $3 \mathrm{G} T \mathrm{x}$ \\
\hline C3P3 & 5.340967 & 5.4942 & 6.746587 & 4.177874 & 4.354117 & 0.797 & 0.208 & 0.549 & 0.502 \\
\hline $\mathrm{C} 4 \mathrm{P} 4$ & 4.976798 & 5.969916 & 5.470431 & 5.611531 & 5.662853 & $0.02 *$ & 0.341 & 0.446 & 0.39 \\
\hline F3C3 & 1.621158 & 2.043488 & 1.706862 & 2.504427 & 1.959637 & 0.186 & 0.878 & 0.447 & 0.572 \\
\hline $\mathrm{F} 4 \mathrm{C} 4$ & 4.020936 & 4.044444 & 1.622194 & 29.3114 & 2.208652 & 0.889 & 0.345 & 0.344 & 0.488 \\
\hline F7T3 & 9.88892 & 11.00887 & 3.207676 & 4.614556 & 3.495417 & 0.442 & 0.306 & 0.42 & 0.343 \\
\hline F8T4 & 3.395488 & 4.485198 & 3.142139 & 4.29439 & 3.514288 & $0.025^{*}$ & 0.826 & 0.68 & 0.941 \\
\hline FP1F3 & 2.691339 & 2.631254 & 0.967207 & 4.522112 & 2.000668 & 0.64 & 0.351 & 0.648 & 0.739 \\
\hline FP1F7 & 2.363549 & 2.473873 & 1.176674 & 4.548404 & 2.088602 & 0.474 & 0.407 & 0.572 & 0.871 \\
\hline FP2F4 & 1.934579 & 1.24225 & 1.256921 & 28.6646 & 1.393871 & 0.376 & 0.363 & 0.317 & 0.529 \\
\hline FP2F8 & 6.065513 & 5.79361 & 2.239224 & 3.744087 & 2.218779 & 0.791 & 0.407 & 0.644 & 0.407 \\
\hline P3O1 & 10.46362 & 10.23068 & 9.782411 & 9.523682 & 9.707432 & 0.742 & 0.741 & 0.77 & 0.776 \\
\hline $\mathrm{P} 4 \mathrm{O} 2$ & 22.36636 & 11.1847 & 4.627215 & 4.566124 & 4.103068 & 0.334 & 0.195 & 0.195 & 0.184 \\
\hline T3T5 & 16.76994 & 18.83357 & 4.138632 & 4.911315 & 3.661646 & 0.232 & 0.329 & 0.362 & 0.322 \\
\hline T4T6 & 3.636873 & 4.050971 & 2.614173 & 3.213311 & 2.758881 & 0.101 & 0.53 & 0.813 & 0.608 \\
\hline T5O1 & 6.184431 & 6.593343 & 5.534869 & 8.119351 & 7.701566 & 0.469 & 0.304 & 0.347 & 0.254 \\
\hline $\mathrm{T} 6 \mathrm{O} 2$ & 17.33462 & 6.211035 & 5.470911 & 4.60255 & 4.563722 & 0.342 & 0.311 & 0.277 & 0.276 \\
\hline
\end{tabular}

*Significant values.

Table 9. Gamma power.

\begin{tabular}{|c|c|c|c|c|c|c|c|c|c|}
\hline \multicolumn{6}{|l|}{ Mean } & \multicolumn{4}{|c|}{ p-Value } \\
\hline Channel & Normal & $2 G R x$ & $2 \mathrm{G} T \mathrm{x}$ & 3G Rx & $3 \mathrm{G} T \mathrm{x}$ & $2 \mathrm{G} T \mathrm{x}$ & $2 \mathrm{G} T \mathrm{x}$ & 3G Rx & $3 \mathrm{G} T \mathrm{x}$ \\
\hline C3P3 & 0.318761 & 0.329435 & 0.343972 & 0.188201 & 0.184634 & 0.841 & 0.477 & 0.433 & 0.339 \\
\hline C4P4 & 0.266914 & 0.341622 & 0.305035 & 0.296963 & 0.276066 & 0.043 & 0.491 & 0.707 & 0.859 \\
\hline F3C3 & 0.071072 & 0.083921 & 0.063345 & 0.124251 & 0.074329 & 0.473 & 0.851 & 0.546 & 0.94 \\
\hline $\mathrm{F} 4 \mathrm{C} 4$ & 0.25874 & 0.167815 & 0.058373 & 1.31363 & 0.098422 & 0.334 & 0.319 & 0.369 & 0.434 \\
\hline F7T3 & 2.156966 & 0.830969 & 0.188178 & 0.26338 & 0.192301 & 0.326 & 0.326 & 0.346 & 0.33 \\
\hline F8T4 & 0.198848 & 0.250706 & 0.157893 & 0.246277 & 0.177174 & 0.185 & 0.68 & 0.778 & 0.858 \\
\hline FP1F3 & 0.171421 & 0.096915 & 0.031164 & 0.304785 & 0.098226 & 0.309 & 0.325 & 0.658 & 0.635 \\
\hline FP1F7 & 0.148836 & 0.106567 & 0.039518 & 0.301814 & 0.099475 & 0.382 & 0.345 & 0.6 & 0.704 \\
\hline FP2F4 & 0.129758 & 0.072329 & 0.058892 & 1.29905 & 0.066483 & 0.432 & 0.302 & 0.32 & 0.41 \\
\hline FP2F8 & 0.402316 & 0.221096 & 0.116385 & 0.222992 & 0.100202 & 0.442 & 0.395 & 0.627 & 0.37 \\
\hline P3O1 & 0.731965 & 0.630013 & 0.61786 & 0.617347 & 0.561138 & 0.125 & 0.418 & 0.674 & 0.407 \\
\hline $\mathrm{P} 4 \mathrm{O} 2$ & 0.93986 & 0.392144 & 0.208449 & 0.203325 & 0.160679 & 0.187 & 0.228 & 0.228 & 0.202 \\
\hline T3T5 & 1.145913 & 0.595157 & 0.205202 & 0.232311 & 0.164414 & 0.36 & 0.337 & 0.352 & 0.323 \\
\hline T4T6 & 0.18442 & 0.171737 & 0.092316 & 0.138098 & 0.10774 & 0.762 & 0.469 & 0.739 & 0.659 \\
\hline T5O1 & 0.411858 & 0.437174 & 0.353302 & 0.53835 & 0.443261 & 0.595 & 0.257 & 0.442 & 0.666 \\
\hline T6O2 & 0.613325 & 0.394024 & 0.342357 & 0.256884 & 0.236904 & 0.415 & 0.313 & 0.191 & 0.173 \\
\hline
\end{tabular}




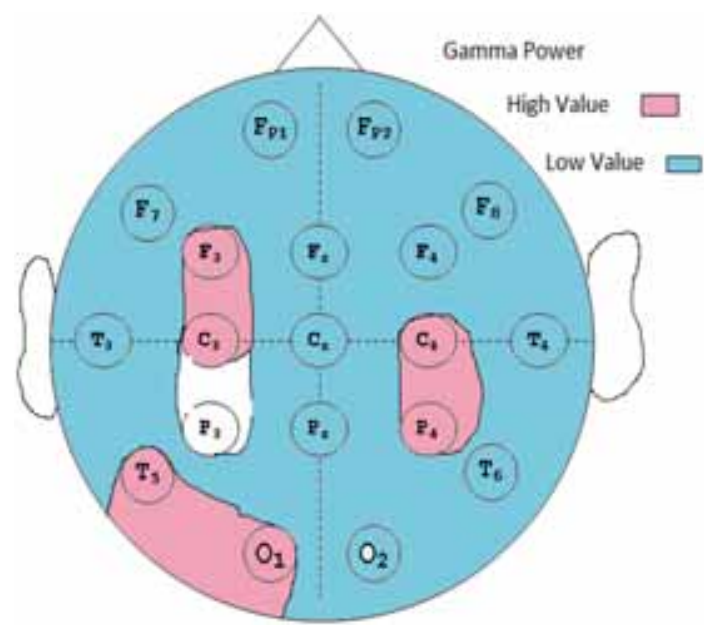

Figure 10. Gamma power channel-wise impact.

T4, F4, F8, FP2, left occipital T5, O1, and left frontal F7, FP1.

The theta power is increased at T4, F8, T5, O1 and F7, FP1. The decrease in the theta power is observed as shown in figure 7.

In the different modes of transmission, significant decrease in theta power is observed at channel P3O1 in 2GRx mode. Otherwise, at other modes, the response to the radiations is mixed and only in $2 \mathrm{GRx}$ mode the theta power is decreased with the radiations as depicted in table 6 . The alpha power has increased at many regions such as T4, T6, C4, P4, F7, FP1, T5, O1, F3 and C3 with significant decrease at $\mathrm{P} 4 \mathrm{O} 2$ in all the modes as shown in table 7. When examined channel-wise, it is observed that there is increase at the right ear lobe side and left occipital side extending to the left frontal as shown in figure 8.

The increase in the alpha wave is the indication of relaxed state which is in agreement with Lee et al [41] who suggested the use of pulsed magnetic field (pMF) as a stimulus to increase the alpha activity. The beta power is depicted in table 8 and figure 9 and has similar behavior as alpha power except at F7 and FP1.

The gamma power has significantly increased at C4P4 in 2GRx mode as depicted in table 9. As shown in figure 10, the gamma power is increased only at $\mathrm{T} 5, \mathrm{O} 1$ at the occipital region and decreased in rest of the part. At T4, F8 i.e., right temporal part and T5, O1, left occipital part have experienced high power due to radiations.

The changes in the brain waves due to RF radiations may bring severe oxidative stress that may cause neurons degeneration [41]. As discussed in [42, 43], the electromagnetic radiations can be used for the treatment of neurodegenerative diseases. Similarly, extensive research on this area can be done and there is a strong belief of the authors that there can also be a ray of hope for coma patient also. Mobile phone radiations come in the non-ionization radiations so they do not cause any genetic changes [44] but other thermal effects cannot be ignored. Sayer [45] reported that an alteration in EEG which can lead to the structural and functional changes in the brain that affects the cognition, mood and behavior.

\section{Conclusion}

The present paper covers an extensive study on the effects on various brain waves due to exposure to mobile radiations. A large data base of 75 healthy subjects is analyzed to study the impact of RF radiations on five brain waves. Significant changes have been observed in almost all the brain waves at the different channels. The changes are observed on the right temporal side towards the right ear to which the mobile phone is held. The left occipital region T5, O1 also showed high values of all parameters measured except Hurst exponent due to these radiations. As seen from the results, there is increase in the values of all the parameters at T4, F8 at the right side of the brain near to the right ear where the mobile phone is held. Also the higher alpha power value is observed at T5, O1 and F7, FP1 channels. It can also been seen that the effect is extending clearly in terms of power and other nonlinear parameters not only the side on which phone is held, but also extending towards the other interior regions of the brain. These parts of the brain are responsible for the emotional memory, verbal understanding, visual processing and attention. In this study, the maximum exposure duration is of 5 min. With this short duration of exposure, visible and measurable variations are observed in all the five brain waves. But in the present time, mainly the youngsters are in extensive use of mobile phones thus may have more adverse effects. Further, clinically supported research is required to quantify such adverse impacts.

\section{Acknowledgements}

The authors are thankful to Dr. Pawan K Kansal, Senior Cardiologist, Mukat Hospital \& Heart Institute, Chandigarh for his valuable suggestions, time and help in the interpretation EEG data. Thanks are due to the learned reviewers for their valuable comments which helped us to improve the quality of the paper.

\section{References}

[1] Kuhn S and Kuster N 2012 Field evaluation of the human exposure from multiband, multisystem mobile phones. IEEE Trans. Electromagn. Compat. 55: 1-13

[2] Mat D A A, Tat F K W, Kipli K, Joseph A, Lias K and Marzuki A S W 2010 Visualization and analytical measurement of electromagnetic radiation from handheld mobile phones. In: Proc. International Conference of Computer Engineering and Applications ICCEA. 246-250 
[3] WHO 2014 Electromagnetic fields and public health: mobile phones. Available: http://www.who.int/mediacentre/fact sheets/fs193/en/ 2011

[4] Gati A, Hadjem A, Wong M F and Wiart J 2009 Exposure induced by WCDMA mobiles phones in operating networks. IEEE Trans. Wirel. Commun. 8: 5723-5727

[5] Perentos N, Croft R J, McKenzie R J and Cosic I 2013 The alpha band of the resting electroencephalogram under pulsed and continuous radio frequency exposures. IEEE Trans. Biomed. Eng. 60: 1702-1710

[6] Bachmann M, Lass J, Kalda J, Säkki M, Tomson R, Tuulik R and Hinrikus H 2006 Integration of differences in EEG analysis reveals changes in human EEG caused by microwave. In: Proc. IEEE Engineering in Medicine and Biology 1597-1600

[7] Hansson M and Lindgren M 2001 Multiple window spectrogram of transient peaks in the electroencephalogram. IEEE Trans Biomed Eng. 48: 284-293

[8] Khandoker A and Pranjape R 2013 Electroencephalogram as a mechanism for human communication. In: Proc. IEEE Canadian Conference of Electrical and Computer Engineering. 1-4

[9] Nandan R and Kaushal S 2014 Analysis of mobile phone radiations effect on beta wave of EEG signal. Int J. Adv. Res. Elect. and Comm. Eng. 3:1854-1860

[10] Mohammed H S, Fahmy H M, Radwan N M and Elsayed A A 2013 Non-thermal continuous and modulated electromagnetic radiation fields effects on sleep EEG of rats. J. Adv. Res. 4: 181-187

[11] Roggeveen S, van J Os, Viechtbauer W and Lousberg R 2015 EEG changes due to experimentally induced $3 \mathrm{G}$ mobile phone radiation. PLoS One 10: 1-10

[12] Isa R M, Pasya I and Taib M N 2012 High frequency brainwaves comparison due to mobile phone radiofrequency emission. In: Proc. Intelligent Systems Modelling and Simulation, ISMS. 191-196

[13] Maby E, Bouquin Jeannès R L and Faucon G 2006 Shortterm effects of GSM mobiles phones on spectral components of the human electroencephalogram. In: Proc. IEEE Engineering in Medicine and Biology 3571-3574

[14] Hinrikus H, Bachmann M, Lass J and Tuulik V 2009 Effect of modulated at different low frequencies microwave radiation on human EEG. Environmentalist 29: 215-219

[15] Smitha C and Narayanan N 2013 Study of brain dynamics under mobile phone radiation using various fractal dimension methods. In: Proc. International Conference on Signal Processing, Image Processing, and Pattern Recognition (ICSIPR). 288-292

[16] Perentos N, Croft R J, Mckenzie R J, Cvetkovic D and Cosic I 2008 The effect of GSM-like ELF radiation on the alpha band of the human resting EEG. IEEE Eng. Med. Biol. Soc. 5680-5683

[17] Isa R M, Pasya I, Taib M N, Jahidin A H, Omar W R W, Fuad N and Norhazman H 2013 EEG brainwave behaviour due to rf exposure using kNN classification. In: Proc. IEEE Conference on System Engineering and Technology. 19-20

[18] Murat Z H and Abdulkadir R S S 2011 The effects of mobile phone usage on human brainwave using EEG. In: Proc. IEEE International Conference on Computer Modelling and Simulation. 36-41

[19] Isa R M, Pasya I, Taib M N, Jahidin A H, Omar W R W and Fuad N 2012 Assessment of brainwave asymmetry and hemisphere dominance due to RF radiation. In: Proc. UKSimAMSS 6th European Modeling Symposium EMS. 153-157

[20] Luis J, Pertega S, Antonio J and Lopez-martin E 2010 Effects of cell-phone radiation on the electroencephalographic spectra of epileptic patients. IEEE Ant. Prop. Mag. 52: 173-179

[21] Vorst A V and Taurisano M D 2000 Experimental thermographic analysis of thermal effects induced on a human head exposed to $900-\mathrm{MHz}$ fields of mobile phones. IEEE Trans. Microw. Theory Tech. 48: 2022-2032

[22] Piuzzi E, Bernardi P, Cavagnaro M, Pisa S and Lin J C 2011 Analysis of adult and child exposure to uniform plane waves at mobile communication systems. IEEE Trans. Electromag. Compatibility 53: 38-47

[23] Lin J C 2010 Acquired cognitive behavior changes in children from cell-phone use health effects. IEEE Microw. Mag. 11: $112-114$

[24] Ilvonen S and Sarvas J 2007 Magnetic-field-induced ELF currents in a human body by the use of a GSM phone. IEEE Trans. Electromagn. Compat. 49: 294-301

[25] Mat D A A, Kho F, Joseph A, Kipli K, Lias K and Wani A S 2010 Electromagnetic radiation from mobile phone near earskull region. In: Proc. International Conference on Computer and Communication Engineering ICCCE. 1-5

[26] Kaur B, Singh S and Kumar J 2015 A Study of SAR pattern in biological tissues due to RF exposure. In: Proc. Recent advances in Engineering and Computational Sciences 1-5

[27] Perentos N, Croft R J, McKenzie R J, Cvetkovic D and Cosic I 2007 Comparison of the effects of continuous and pulsed mobile phone like RF exposure on the human EEG. Australas. Phys. Eng. Sci. Med. 30: 274-280

[28] Lin J C 2003 Cellular telephone radiation and electroencephalograms (EEG) of the human brain. IEEE Antennas Propag. Mag. 45:150-153

[29] Danker-hopfe H, Dorn H and Bahr A 2011 Mobile phones and sleep effects of electromagnetic fields emitted by mobile phones (GSM 900 and WCDMA/UMTS) on the macrostructure of sleep. J. Sleep Res. 73-81

[30] Murat Z H, Taib M N, Kadir R S S A, Jahidin A H, Lias S and Isa R M 2011 Comparison between the left and the right brainwaves for delta and theta frequency band after horizontal rotation intervention. In: Proc. Computational Intelligence, Communication Systems and Networks, 368-372

[31] Tyagi A, Duhan M and Bhatia D 2011 Effect of mobile phone radiation on brain activity GSM vs CDMA power. Int. J. Sc. Tech. Mgmt. 2: 1-5

[32] Zhang A and Xie Y 2014 Chaos theory-based data-mining technique for image endmember extraction: Laypunov index and correlation dimension (L and D). IEEE Trans. Geosci. Remote Sens. 52: 1935-1947

[33] Jingla L I, Jin W, Guozhen G U O, Yao G U O and Zhong J L W 2003 Multichannel EEG in freely moving rats. In: Proc. AsiaPacific Conference on Environmental Electromagnetics. 134-137

[34] Karmakar C, Udhayakumar R K and Palaniswami M 2015 Distriution Entropy (DistEn): A complexity measure to detect arrhythmia from short length RR interval time series. In: Proc. IEEE Eng. Med. Biol. Soc. EMBS. 5207-5210

[35] Lu S L S, Chen X C X, Kanters J K, Solomon I C and Chon K H 2008 Automatic selection of the threshold value for approximate entropy. IEEE Trans. Biomed. Eng. 55:1966-1972 
[36] Zang X and Howell J 2003 Correlation dimension and lyapunov exponent based isolation of plant-wide oscillations. German Arts and Letters 8-13

[37] Bermudez G R and Laencina P J G 2015 Analysis of EEG signals using nonlinear dynamics and chaos: A review. Appl. Math. Inf. Sci. 9: 2309-2321

[38] https://www.spss-tutorials.com/basics/

[39] https://www.spss-tutorials.com/statistical-significance/

[40] Hornero R, Abásolo D, Escudero J and Gómez C 2009 Nonlinear analysis of electroencephalogram and magnetoencephalogram recordings in patients with Alzheimer's disease. Phil. Trans. R. Soc. A. 317-336

[41] Lee H S, Kim S and Hwang D G 2011 Effects of pulsed magnetic field stimulus on electroencephalogram alpha activity. IEEE Trans. Magnetics 47: 3060-3062
[42] Mesiti F, Anders P, Na A and Balasingham I 2012 Nano communication networks on the modeling and analysis of the RF exposure on biological systems: A potential treatment strategy for neurodegenerative diseases. Nano Commun. Netw. 3:103-115

[43] Khaleghi A, Sendi M S E, Chávez-Santiago R, Mesiti F and Balasingham I 2012 Exposure of the human brain to an electromagnetic plane wave in the $100-1000 \mathrm{MHz}$ frequency range for potential treatment of neurodegenerative diseases. IET Microwaves, Antennas Propag. 6:1565-1572

[44] Kshetrimayum R S 2008 Mobile Phones: Bad for your Health?. IEEE Potentials. 1820

[45] Sayer J 2015 Brain wave warping effect of mobile phones, Study reveals. http://www.greenmedinfo.com/blog/brainwave-warping-effect-mobile-phones-study-reveals 